Revista Brasil. Bot., V.31, n.4, p.587-596, out.-dez. 2008

\title{
Micromorfologia da superfície do aquênio em Bulbostylis Kunth (Cyperaceae) ${ }^{1}$
}

\author{
ANA PAULA DO NASCIMENTO PRATA ${ }^{2,5}$, WILLIAM WAYT THOMAS ${ }^{3}$ e \\ MARIA DAS GRAÇAS LAPA WANDERLEY ${ }^{4}$
}

(recebido: 20 de dezembro de 2007; aceito: 07 de agosto de 2008)

\begin{abstract}
Micromorphology of achene surface in Bulbostylis Kunth (Cyperaceae)). Bulbostylis comprises approximately 150 species distributed in the tropics and subtropics of both hemispheres. The morphology of the achene surface, using scanning electron microscopy, was used to differentiate 38 species of Bulbostylis Kunth (Cyperaceae) and revealed the presence of three patterns of ornamentation on the achene surface: tuberculate, reticulate, and transversely rugose. Other characters of taxonomic interest were also observed, including the primary sculpturing, the ornamentation of the anticlinal cell walls of the epidermis, the presence or absence of silica bodies, and the shape of the epidermal cells.
\end{abstract}

Key words - achene, Bulbostylis, Cyperaceae, morphology, systematics

RESUMO - (Micromorfologia da superfície do aquênio em Bulbostylis Kunth (Cyperaceae)) Bulbostylis possui aproximadamente 150 espécies, distribuídas nas regiões tropicais e subtropicais de ambos os hemisférios. Caracteres morfológicos da superfície do aquênio, em microscopia eletrônica de varredura, foram utilizados para diferenciar 38 das 45 espécies de Bulbostylis Kunth (Cyperaceae) ocorrentes no Brasil, permitindo o reconhecimento de três padrões de ornamentação da superfície do aquênio: tuberculado, reticulado e transversalmente rugoso. Além destes, outros caracteres de interesse taxonômico foram observados, tais como a variação na escultura primária do aquênio, a ornamentação das paredes anticlinais, a presença ou ausência de corpos silicosos e o contorno das células epidérmicas.

Palavras-chave - aquênio, Bulbostylis, Cyperaceae, morfologia, sistemática

\section{Introdução}

O uso de caracteres morfológicos da superfície dos frutos e sementes é, em geral, bastante significativo na taxonomia das Angiospermas (Denton 1983, Ritter \& Miotto 2006, Zech \& Wujeck 1990). Em Cyperaceae, a utilização de dados provenientes da superfície do fruto torna-se importante para a taxonomia, devido à escassez de caracteres diagnósticos florais e vegetativos (Menapace 1990).

Análises microestruturais da epiderme em diferentes órgãos como folhas, flores e frutos em vários gêneros de Cyperaceae foram realizadas, fornecendo caracteres de importância taxonômica (Govindarajalu 1966, Kukkonen 1967, Metcalfe 1971, Toivonen \& Timonen 1976, Denton 1983).

\footnotetext{
1. Parte da tese de Doutorado do primeiro autor, Programa de Pósgraduação em Botânica da Universidade de São Paulo, São Paulo.

2. Universidade Federal de Sergipe, Departamento de Biologia Campus Universitário Professor Aloísio de Campos, 49100-000 São Cristóvão, Sergipe, Brasil.

3. The New York Botanical Garden, Bronx, 10458-5126, New York, EUA.

4. Instituto de Botânica, Herbário, Caixa Postal 3005, 01061-970 São Paulo, SP, Brasil.

5. Autor para correspondência: apprata@yahoo.com.br
}

O fruto em Cyperaceae é um aquênio (Denton 1978, Menapace 1990, Araújo \& Longhi-Wagner 1997, Lye 2000), embora já tenha sido denominado anteriormente como noz (Persoon 1805, 1807) ou cariopse (Nees 1834, Endlicher 1836). Dos trabalhos que abordam aspectos relacionados ao aquênio dessa família, destaca-se o de Lye (2000) que analisou a morfologia e as funções de várias estruturas dos aquênios de cerca de 600 espécies. Neste trabalho foram apresentados forma, tamanho, peso e coloração dos aquênios, estrutura e anatomia do pericarpo, além da presença de apêndices. $\mathrm{O}$ autor citou que apesar da similaridade existente no aquênio de alguns gêneros de Cyperaceae (particularmente Cyperus e Carex), muitas espécies da família podem ser identificadas através da análise deste órgão.

Dentre os gêneros de Cyperaceae, Carex é um dos mais estudados e o uso da microscopia eletrônica de varredura (MEV) no estudo da epiderme do aquênio demonstrou bons resultados para auxiliar na taxonomia desse gênero, tanto em nível de seção quanto de espécie (Toivonen \& Timonen 1976, Standley 1985, Waterway 1990).

Outros gêneros de Cyperaceae como Cyperus (Denton 1983, Araújo \& Longhi-Wagner 1997), Eleocharis (Menapace 1990) e Scirpus (Schuyler 1971), dentre outros, também tiveram seus frutos analisados 
com o objetivo de testar agrupamentos taxonômicos ou auxiliar na descoberta de novos caracteres que pudessem ajudar na identificação e na elucidação das relações entre as espécies.

Alguns caracteres da superfície do aquênio, como a escultura primária (arranjo e forma das células das paredes anticlinais, o contorno do lúmen celular e a presença ou ausência de corpos de sílica), a escultura secundária (relevo das paredes periclinais externas) e a escultura terciária (secreções epicuticulares) foram observados e ressaltados por Barthlott (1984) e Menapace (1990) como úteis para a taxonomia das Cyperaceae.

Em relação ao estudo do aquênio de representantes brasileiros de Cyperaceae, destacam-se os trabalhos de Oliveira (1980) e Araújo \& Longhi-Wagner (1997). No primeiro, a autora levando em consideração as estruturas florais persistentes no fruto, agrupa os aquênios de 31 gêneros (incluindo Bulbostylis), em cinco tipos: nus, perigínicos, estilínicos, mistos e utriculados. No segundo trabalho, as autoras analisaram os aquênios de 14 táxons pertencentes a Cyperus subg. Anosporum ocorrentes no Rio Grande do Sul. Esta análise forneceu caracteres diagnósticos em nível específico, sendo os referentes à escultura primária destacados, tais como a ornamentação das paredes anticlinais e a presença de corpos silicosos.

Bulbostylis, um gênero tipicamente herbáceo, é caracterizado pela base do estilete espessada e persistente no aquênio (estilopódio) além da ocorrência de tricomas no ápice da bainha foliar. Possui aproximadamente 150 espécies em regiões tropicais a subtropicais, sendo particularmente abundante na América do Sul e África. No Brasil ocorrem cerca de 45 espécies, das quais 16 são endêmicas (Prata 2004). Habitam preferencialmente as áreas quentes e secas, abertas e com muita luminosidade.

A identificação das espécies de Bulbostylis é, em geral, bastante dificultada devido aos seguintes aspectos: extrema redução floral característica da família, notável similaridade morfológica dos órgãos vegetativos, além da carência de descrições completas que reflitam as diferenças morfológicas entre os táxons. Diante disso, é evidente a necessidade de buscar caracteres florais que possam auxiliar na identificação das espécies do gênero.

Apesar das características do aquênio serem consideradas importantes para a identificação das Cyperaceae, com exceção do trabalho de Lye (2000), poucos gêneros desta família foram estudados utilizandose o microscópio eletrônico de varredura. Para o gênero Bulbostylis esse tipo de estudo também é escasso, destacando-se apenas alguns artigos sobre a descrição de novas espécies (Lye 1986, Prata et al. 2007) ou trabalhos de atualizações nomenclaturais (López 2006, López et al. 2007).

O presente trabalho teve como proposta delinear os padrões de ornamentação da superfície do aquênio em Bulbostylis, criando parâmetros que sirvam de subsídios à taxonomia e, dessa forma, acrescentar informações ao conhecimento do gênero.

\section{Material e métodos}

As análises micromorfológicas foram desenvolvidas no Instituto de Botânica (IBt) e no Instituto de Biociências da Universidade de São Paulo (USP). Aquênios maduros de 38 espécies de Bulbostylis (tabela 1), foram analisados por meio de observação direta da superfície do aquênio e da base do estilete persistente, sendo 21 espécies documentadas (figuras 1-27). Foram selecionados dois espécimens de cada táxon, utilizando-se de quatro a seis frutos por exemplar. Os mesmos foram montados diretamente em stubs, sem prétratamento, fixados através de fita dupla face, metalizados com ouro e fotografados em MEV Stereoscan 600, com voltagem entre 15 e $25 \mathrm{~kW}$, em escalas de 200 a 4.000x. A terminologia adotada neste trabalho seguiu a proposta de Barthlott (1984). O termo estilopódio é empregado neste estudo para a base do estilete persistente no aquênio de Bulbostylis (Prata 2004).Os acrônimos das instituições estão de acordo com Holmgren et al. (1990).

Tabela 1. Lista de material examinado de Bulbostylis.

Table 1. Analyzed species, vouchers and Herbarium acrononym.

\begin{tabular}{llc}
\hline Espécie & Coletor & Herbário \\
\hline B. amambayensis Barros & G. Gehrt 3652 & $\mathrm{SP}$ \\
B. brevifolia Palla & J. Segadas-Vianna 2679 & $\mathrm{R}$ \\
B. capillaris (L.) C. B. Clarke & H. Hashimoto 19976 & $\mathrm{SP}$ \\
B. conifera Kunth) C. B. Clarke & J.S. Costa 1207 & $\mathrm{SP}$ \\
B. consanguinea (Kunth) C. B. Clarke & M. Batalha \& W. Mantovani 60 & $\mathrm{SP}$
\end{tabular}


continuação

\begin{tabular}{|c|c|c|}
\hline Espécie & Coletor & Herbário \\
\hline B. conspicua (Boeck.) H. Pfeiff. & G. Hatschbach et al. 28027 & SPF \\
\hline B. distichoides Lye & A.L. Costa \& G.M. Barroso s.n. & ALC 07250 \\
\hline B. edwalliana (Boeck.) Prata \& M. G. López & A. Loefgren \& G. Edwall 2038 & SP \\
\hline B. eleocharoides Kral \& M. Strong & A.P. Prata et al. 1145 & SP \\
\hline B. emmerichiae T. Koyama & R.C. Mendonça \& D. Alvarenga 625 & IBGE \\
\hline B. fasciculata Uittien & A.P. Prata et al. 903 & SP \\
\hline B. fendleri C. B. Clarke & S.M. Campos 107 & SP \\
\hline B. fimbriata (Nees) C. B. Clarke & M.R. Marques-Leitão et al. s.n. & $\mathrm{BHCB}$ \\
\hline B. hirtella (Schrad.) Urb. & M. Sakane 589 & SP \\
\hline B. jacobinae (Steud.) Lindm. & A.P. Prata et al. 1054 & SP \\
\hline B. junciformis (Kunth) C. B. Clarke & W. Mantovani 47 & SP \\
\hline B. juncoides (Vahl) Kuk & L.T. Dombrowski 7691 & MBM \\
\hline B. lagoensis (Boeck.) Prata \& M. G. López & A. Loefgren 1091 & SP \\
\hline B. lanata (Kunth) Lindm. & A.P. Prata 515 & SP \\
\hline B. latifolia Kral \& M. Strong & M.G. Wanderley 1877 \& R. Kral 75909 & SP \\
\hline B. loefgrenii (Boeck.) Prata \& López & A. Loefgren 976 & SP \\
\hline B. lombardii Kral \& M. Strong & A.B. Joly \& J. Semir 2873 & UEC \\
\hline B. major Palla & C. Novaes s.n. & SP \\
\hline B. nesiotis (Hemsl.) C. B. Clarke & P.C. Porto 8170 & $\mathrm{RB}$ \\
\hline B. pachypoda Kral \& M. Strong & R.M. Harley 10512 & NY \\
\hline B. paradoxa (Spreng.) Lindm. & E.P. Heringer et al. 5361 & IBGE \\
\hline B. paraensis C. B. Clarke & G. Hashimoto 19974 & SP \\
\hline B. scabra (Presl.) C. B. Clarke & F.C. Hoehne 17551 & SP \\
\hline B. schomburgkiana (Steud.) M. Strong & A.P. Prata et al. 488 & SP \\
\hline B. sellowiana (Kunth) Palla & V.C. Souza et al. 8779 & SPF \\
\hline B. smithii Barros & L.B. Smith et al. 6889 & NY \\
\hline B. sphaerocephala (Boeck.) C. B. Clarke & M. Batalha \& W. Mantovani 105 & SP \\
\hline B. splendens M. Strong & I. Cordeiro 241 & SP \\
\hline B. svensoniana Steyermark & F.A. Vitta 230 & SP \\
\hline B. tenuifolia (Rudge.) J. F. Macbr. & G. Davidse \& W.G. Darcy 11792 & SP \\
\hline B. truncata (Nees) M. Strong & I. Mimura 111 & SP \\
\hline B. vestita (Kunth) C. B. Clarke & M. Lисеп̃o $235 b$ & UFP \\
\hline B. sp. nov. & A.P. Prata 859 & SP \\
\hline
\end{tabular}

Neste estudo com o MEV, foram observados, além do contorno do aquênio, os seguintes caracteres referentes à estrutura primária: forma e orientação das células epidérmicas, ornamentação da parede anticlinal e presença ou ausência de corpos silicosos.

\section{Resultados e discussão}

Segundo Oliveira (1980), o aquênio de Bulbostylis enquadra-se no tipo estilínico, no qual apenas a base do estilete é persistente no aquênio, na forma de tubérculo, caliptra ou rostro.

A maioria das espécies estudadas apresenta aquênio trígono (figuras 1-3, 15-22, 24-27,) e estilete trífido. O contorno do aquênio de $B$. lanata ao MEV apresenta-se biconvexo (figura 4), contudo, na descrição original da espécie (Kunth 1816) consta aquênio trígono e estilete trífido. Já $B$. smithii apresenta aquênio biconvexo e estilete bífido (figura 5). Na descrição original desta espécie (Barros 1959) consta estilete bífido, mas não traz informações quanto a secção transversal do mesmo.

Cerca de $75 \%$ das espécies apresentadas possuem contorno do aquênio obovóide. O restante das espécies possui aquênio com diferentes formas: obcônico, piriforme e obpiriforme (tabela 2).

Foi observada em 28 das espécies analisadas a presença de ângulo frontal espessado e com ornamentação diferenciada das demais partes do aquênio (figuras 19, 24-27), exceto em B. amambayensis, B. arenaria, $B$. capillaris (geralmente), B. carajana, B. conspicua, $B$. distichoides, B. eleocharoides, B. fasciculata, B. jacobinae, B. junciformis, B. lagoensis, B. loefgrenii, B. smithii, $B$. 
Tabela 2. Informações morfológicas, padrões de ornamentação da superfície dos aquênios e ocorrência no Brasil das espécies de Bulbostylis analisadas. (POA = Padrão de ornamentação do aquênio: $\mathrm{R}=$ reticulado, $\mathrm{TR}=$ transversalmente rugoso, $\mathrm{T}=$ tuberculado. $\mathrm{TI}=$ Tipo de inflorescência: ant $=$ antelóide, cap $=$ capitada, uni $=$ uniespiculada $. \mathrm{CA}=$ Contorno do aquênio: obo $=$ obovóide, obc $=$ obcônico, pir $=$ piriforme, obo a pir $=$ obovóide a piriforme $. \mathrm{CE}=$ Caracteres do estilopódio: $\mathrm{P}=$ persistente, $\mathrm{D}=$ decíduo. $\mathrm{CS}=$ Corpos silicosos: $\mathrm{PR}=$ presente, $\mathrm{A}=$ ausente. $\mathrm{O}=$ Ocorrência: $\mathrm{N}=$ Norte, $\mathrm{NE}=$ Nordeste, $\mathrm{CE}=$ Centro-oeste, $\mathrm{S}=\mathrm{Sul}, \mathrm{SE}=$ Sudeste).

Table 2. Analyzed species, morphologic informations, ornamentation patterns of achene surface and occurrence in Brazil. $(\mathrm{POA}=$ Ornamentation patterns of achene: $\mathrm{R}=$ reticulate, $\mathrm{TR}=$ transversely rugose, $\mathrm{T}=$ tuberculate. $\mathrm{TI}=$ Inflorescence type: ant $=$ anthelate, cap $=$ capitate, uni $=$ uniespiculate. $\mathrm{CA}=$ Achene shape: obo $=$ obovoid, obc $=$ obconic, pir $=$ pyriform, obo a pir = obovoid to pyriform. $\mathrm{CE}=$ Characteres of stylopodium: $\mathrm{P}=$ persistent, $\mathrm{D}=$ deciduous. $\mathrm{CS}=$ silica body: $\mathrm{PR}=$ present, $\mathrm{A}=$ absent. $\mathrm{O}=$ Occurence: $\mathrm{N}=$ North, $\mathrm{NE}=$ Northeast, $\mathrm{CE}=$ Central west, $\mathrm{S}=$ South, $\mathrm{SE}=$ Southeast).

\begin{tabular}{|c|c|c|c|c|c|c|}
\hline Espécie & POA & TI & $\mathrm{CA}$ & $\mathrm{CE}$ & $\mathrm{CS}$ & $\mathrm{O}$ \\
\hline 1. B. amambayensis & $\mathrm{R}$ & cap & obo & $\mathrm{P}$ & A & N,CE,SE \\
\hline 2. B. brevifolia & $\mathrm{TR}$ & ant & obo & $\mathrm{P}$ & PR & $\mathrm{NE}$ \\
\hline 3. B. capillaris & $\mathrm{T}$ & ant & obo & $\mathrm{P}$ & PR & NE,SE,S \\
\hline 4. B. conifera & TR & uni & obo & $\mathrm{P}$ & PR & N,NE,CE,SE \\
\hline 5. B. consanguinea & $\mathrm{T}$ & ante & obo & $\mathrm{P}$ & PR & N,NE,CE,SE \\
\hline 6. B. conspicua & TR & ante & obo & $\mathrm{P}$ & PR & CE,SE \\
\hline 7. B. distichoides & $\mathrm{T}$ & cap & obo & $\mathrm{P}$ & PR & $\mathrm{NE}$ \\
\hline 8. B. edwalliana & $\mathrm{T}$ & ant & obo & $\mathrm{P}$ & PR & $\mathrm{CE}, \mathrm{SE}$ \\
\hline 9. B. eleocharoides & $\mathrm{T}$ & uni & obo & $\mathrm{P}$ & PR & CE,SE \\
\hline 10. B. emmerichiae & TR & capi & pir & $\mathrm{D}$ & A & NE, CE,SE \\
\hline 11. B. fasciculata & $\mathrm{R}$ & ant & obo & $\mathrm{P}$ & $\mathrm{A}$ & NE, CE,SE \\
\hline 12. B. fendleri & $\mathrm{T}$ & ant & obo & $P$ & PR & $\mathrm{N}, \mathrm{CE}, \mathrm{S}$ \\
\hline 13. B. fimbriata & $\mathrm{R}$ & cap & obo & $\mathrm{P}$ & PR & NE, CE,SE, S \\
\hline 14. B. hirtella & $\mathrm{T}$ & ant & obc & $\mathrm{P}$ & $\mathrm{PR}$ & $\mathrm{NE}, \mathrm{CE}, \mathrm{SE}, \mathrm{S}$ \\
\hline 15. B. jacobinae & $\mathrm{T}$ & ant & obc & $\mathrm{P}$ & PR & NE, CE, SE, S \\
\hline 16. B. junciformis & $\mathrm{R}$ & ant & obc & $\mathrm{P}$ & $\mathrm{A}$ & NE, CE, SE, S \\
\hline 17. B. juncoides & TR & cap & obo & $\mathrm{P}$ & PR & $\mathrm{S}$ \\
\hline 18. B. lagoensis & $\mathrm{T}$ & ant & obo & $\mathrm{P}$ & PR & NE, CE, SE \\
\hline 19. B. lanata & $\mathrm{R}$ & uni & obo & $\mathrm{P}$ & A & $\mathrm{N}, \mathrm{NE}$ \\
\hline 20. B. latifolia & $\mathrm{T}$ & ant & obo & $\mathrm{P}$ & PR & $\mathrm{CE}$ \\
\hline 21. B. loefgrenii & $\mathrm{R}$ & ant & obo & $\mathrm{P}$ & $\mathrm{A}$ & SE \\
\hline 22. B. lombardii & TR & cap & obp & $\mathrm{P}$ & PR & SE \\
\hline 23. B. major & $\mathrm{T}$ & ant & obo & $\mathrm{P}$ & PR & SE \\
\hline 24. B. nesiotis & $\mathrm{TR}$ & cap & obo & $\mathrm{P}$ & $\mathrm{PR}$ & $\mathrm{SE}$ \\
\hline 25. B. pachypoda & $\mathrm{R}$ & cap & obo & $\mathrm{P}$ & A & $\mathrm{CE}, \mathrm{SE}$ \\
\hline 26. B. paradoxa & $\mathrm{TR}$ & uni & obo a pir & $\mathrm{P}$ & A & $\mathrm{N}, \mathrm{NE}, \mathrm{CE}, \mathrm{SE}, \mathrm{S}$ \\
\hline 27. B. paraensis & $\mathrm{T}$ & ant & obo & $\mathrm{P}$ & PR & $\mathrm{N}, \mathrm{CE}, \mathrm{SE}$ \\
\hline 28. B. scabra & $\mathrm{T}$ & ant & obc & $\mathrm{P}$ & $\mathrm{A}$ & NE, CE, SE, S \\
\hline 29. B. schomburgkiana & TR & ant & obo & $\mathrm{P}$ & A & $\mathrm{N}$ \\
\hline 30. B. sellowiana & $\mathrm{TR}$ & cap & obc & $\mathrm{D}$ & A & NE, CE, SE, S \\
\hline 31. B. smithii & $\mathrm{TR}$ & ant & obc & $\mathrm{P}$ & A & SE \\
\hline 32. B. sphaerocephala & $\mathrm{R}$ & cap & obo & $\mathrm{P}$ & PR & CE, SE, S \\
\hline 33. B. splendens & $\mathrm{T}$ & uni & obo & $\mathrm{P}$ & A & $\mathrm{N}$ \\
\hline 34. B. svensoniana & $\mathrm{T}$ & ant & obo & $\mathrm{P}$ & A & CE,SE \\
\hline 35. B. tenuifolia & $\mathrm{R}$ & ant & obo & $\mathrm{P}$ & $\mathrm{A}$ & $\mathrm{N}, \mathrm{SE}$ \\
\hline 36. B. truncata & $\mathrm{T}$ & ant & obc & $\mathrm{P}$ & PR & N, NE, SE \\
\hline 37. B. vestita & $\mathrm{T}$ & ant & obo & $\mathrm{P}$ & PR & $\mathrm{N}, \mathrm{NE}$ \\
\hline 38. B. sp nov. & $\mathrm{TR}$ & ant & obo & $\mathrm{P}$ & PR & SE, S \\
\hline
\end{tabular}



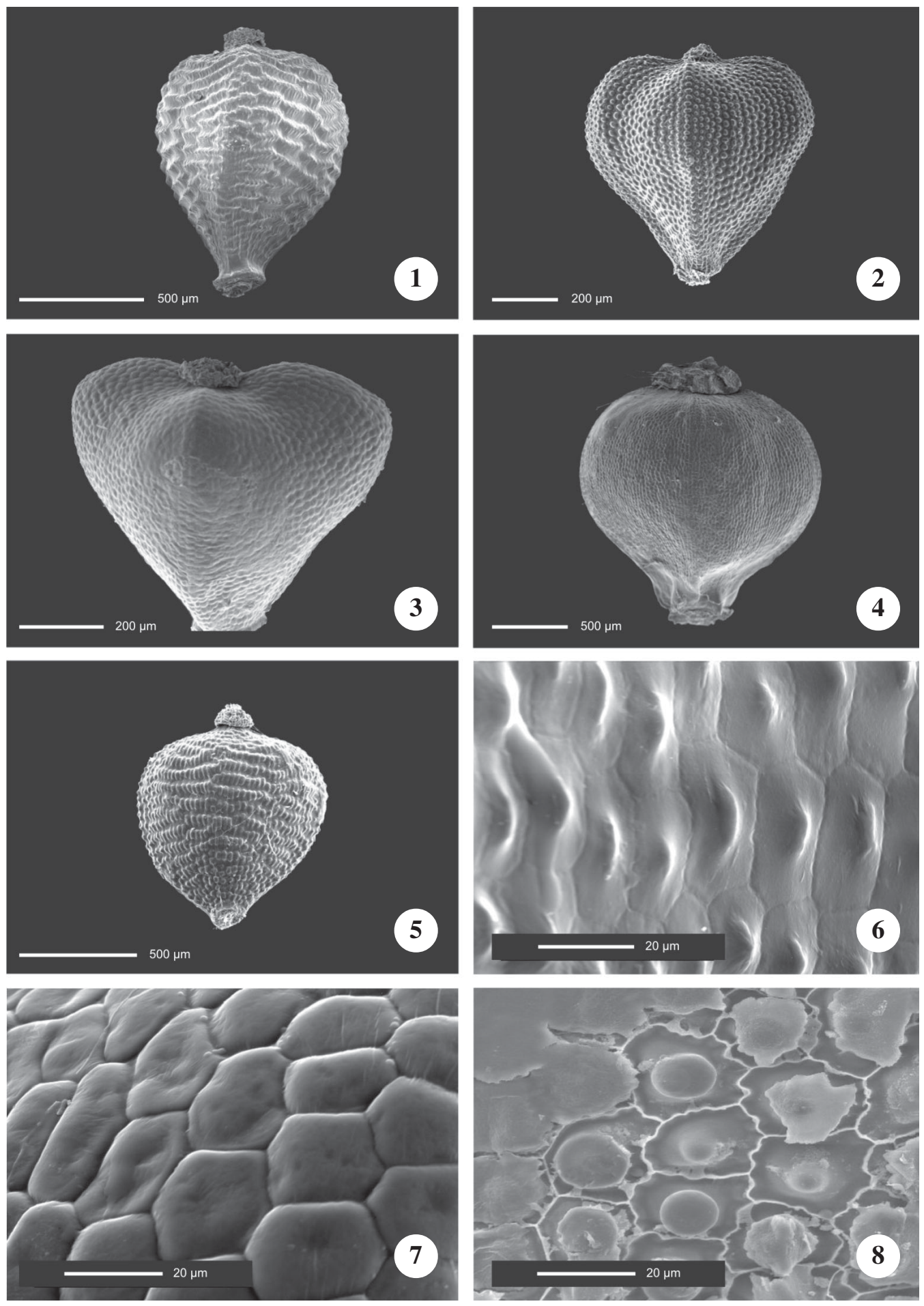

Figuras 1-8. Aquênio das espécies de Bulbostylis ao Microscópio Eletrônico de Varredura. 1. Bulbostylis sp. nov. 2. B. vestita. 3. B. truncata. 4. B. lanata. 5. B. smithii. 6. B. lagoensis: detalhe da superfície. 7. B. truncata: detalhe da superfície. 8. B. jacobinae: detalhe da superfície.

Figure 1-8. Achene surface of Bulbostylis species to the Scanning Electron Microscope. 1. Bulbostylis sp. nov. 2. B. vestita. 3. B. truncata. 4. B. lanata. 5. B. smithii. 6. B. lagoensis: surface detail. 7. B. truncata: surface detail. 8. B. jacobinae: surface detail. 

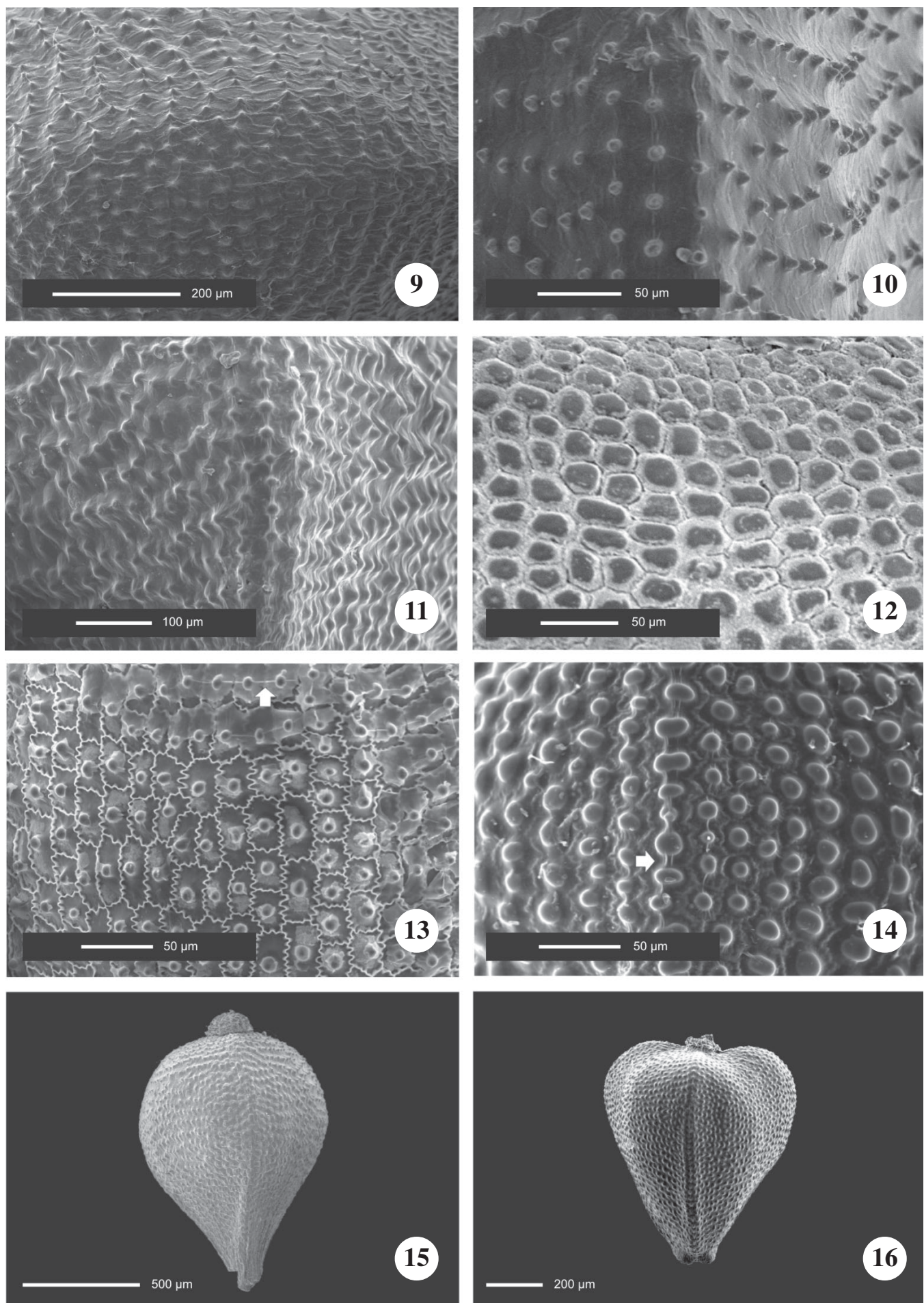

Figuras 9-16. Aquênio das espécies de Bulbostylis ao Microscópio Eletrônico de Varredura. 9. B. capillaris: detalhe da superfície. 10. B. lagoensis: detalhe da superfície. 11. B. consanguinea: detalhe da superfície. 12. B. junciformis. 13. B. nesiotis: detalhe da superfície. 14. B. vestita: detalhe da superfície. 15. B. capillaris. 16. B. latifolia.

Figure 9-16. Achene surface of Bulbostylis species to the Scanning Electron Microscope. 9. B. capillaris: surface detail. 10. B. lagoensis: surface detail. 11. B. consanguinea: surface detail. 12. B. junciformis. 13. B. nesiotis: surface detail. 14. B. vestita: surface detail. 15. B. capillaris. 16. B. latifolia. 
sphaerocephala e $B$. splendens, espécies onde não foram observadas nenhuma diferenciação em espessura ou ornamentação em relação as demais partes do aquênio.

De acordo com Lye (2000), a parede anticlinal das células epidérmicas nas Cyperaceae é reta ou fraca a fortemente sinuosa. Para a maioria das espécies analisadas de Bulbostylis, as paredes anticlinais são retas (figuras 6, 7), exceto em B. amambayensis, B. jacobinae (figura 8), B. fimbriata, B. major, B. nesiotis, B. scabra e $B$. vestita, que são sinuosas. Resultado semelhante foi obtido por Araujo \& Longhi-Wagner (1997) para alguns taxa pertencentes ao gênero Cyperus subg. Anosporum (Nees) C.B. Clarke ocorrentes no Rio Grande do Sul.

As células epidérmicas dos aquênios em Cyperaceae são geralmente isodiamétricas, arredondadas, ou mais frequentemente irregularmente hexagonais (Lye 2000). Nas espécies analisadas de Bulbostylis estas células possuem 5-6 faces (figuras 6-8), tendo sido considerado um caráter comum para o gênero em estudo.

Corpos silicosos foram observados na parede periclinal externa da maioria dos táxons estudados (figuras 8-11, 13, 14, 23, tabela 2), sendo ausentes em B. amambayensis, B. eleocharoides, B. emmerichiae, $B$. fasciculata, B. graminifolia, B. junciformis (figura 12), B. lanata, B. loefgrenii, B. pachypoda, B. paradoxa, B. scabra, B. schomburgkiana, B. sellowiana, B. smithii, $B$. splendens, $B$. svensoniana e $B$. tenuifolia. Conexões entre os corpos silicosos e as paredes anticlinais internas foram observadas apenas em $B$. nesiotis, B. splendens e $B$. vestita (figuras 13-14). Araújo \& Longhi-Wagner citaram a presença de conexões em algumas espécies de Cyperus.

Ressaltamos que em algumas espécies pode haver uma diferença de ornamentação da superfície do aquênio quanto à presença, ausência ou formato dos corpos de sílica, se a análise for realizada com a presença da parede periclinal externa e sem parede periclinal externa (López com. pess.). Em B. loefgrenii, B. junciformis e B. svensoniana não foram observados corpos silicosos na parede periclinal externa. Entretanto, López (com. pess.), analisando aquênios com e sem parede periclinal externa, cita a presença de corpos silicosos nestas espécies, após a retirada da parede periclinal. Destacamos que esta diferença nos resultados deve-se ao uso de diferentes metodologias e que estudos mais aprofundados com estas espécies são necessários para descrever com maior precisão esta característica.

A organização das células epidérmicas determina diferentes padrões de ornamentação da superfície do fruto. Quando as células epidérmicas estão alinhadas em fileiras longitudinais e as células adjacentes se alternam uma com as outras ou estão dispostas transversalmente, a superfície do aquênio é descrita como reticulada ou longitudinalmente estriada. Quando o aquênio é formado por células alongadas longitudinalmente e com rugas transversais proeminentes, o aquênio é dito rugoso ou transversalmente rugoso (Lye 2000).

As espécies de Bulbostylis analisadas neste trabalho apresentaram três padrões de ornamentação da superfície do aquênio: tuberculado $(\mathrm{T})$, reticulado $(\mathrm{R})$ e transversalmente rugoso (TR) (tabela 2). Estes padrões se mostraram constantes na mesma espécie e possuem importância taxonômica.

No primeiro padrão, podemos observar a presença de corpos de sílica distribuídos em toda a extensão do aquênio, mas sem formar linhas transversais (figuras 2, $3,15,16,21,22,27)$. No padrão reticulado, os corpos de sílica são ausentes. Nota-se apenas o contorno celular, parecendo um retículo, sem nenhuma protuberância visível (figuras $4,12,17,18$ ). Ao observarmos a ornamentação do aquênio de algumas espécies, como, por exemplo, em B. fasciculata, ao microscópio óptico (Prata 2004), podemos notar a presença de algumas linhas ou retículos não definidos. Em geral esses aquênios são referidos como lisos ou reticulados. No terceiro padrão, na maioria das vezes, notamos a presença dos corpos de sílica, além do fato das células estarem organizadas transversalmente semelhantes a rugas (figuras 1, 5, 19, 20, 24-26). Nota-se, na maioria das espécies com este padrão, o desnível das rugas em relação ao restante do aquênio. Essas rugas podem ser mais ou menos proeminente de acordo com a organização das células epidérmicas de cada espécie.

De acordo com Lye (2000), a superfície do aquênio de muitos gêneros de Cyperaceae é ornamentada por papilas (Cyperus, algumas espécies de Bulbostylis), tubérculos (Abildgaardia ovata (Burm. f.) Kral, várias espécies de Fimbristylis, Scleria e Rhynchospora rubra (Lour.) T. Maquino, rugas ou vários apêndices como glândulas ou tricomas. Entretanto, este tipo de ornamentação ou apêndice não foi observado nos táxons analisados neste trabalho. Acredita-se que este tipo de ornamentação em Bulbostylis deva ocorrer talvez apenas nas espécies africanas citadas por Lye (2000).

Os padrões de ornamentação da superfície do aquênio tuberculado e transversalmente rugoso foram encontrados na maioria das espécies de Bulbostylis analisadas (17 e 12 spp., respectivamente, tabela 2). Os demais táxons apresentam superfície reticulada.

Ao se fazer uma correlação entre o tipo de inflorescência das espécies e o padrão de ornamentação da superfície do aquênio, concluímos que entre as cinco 

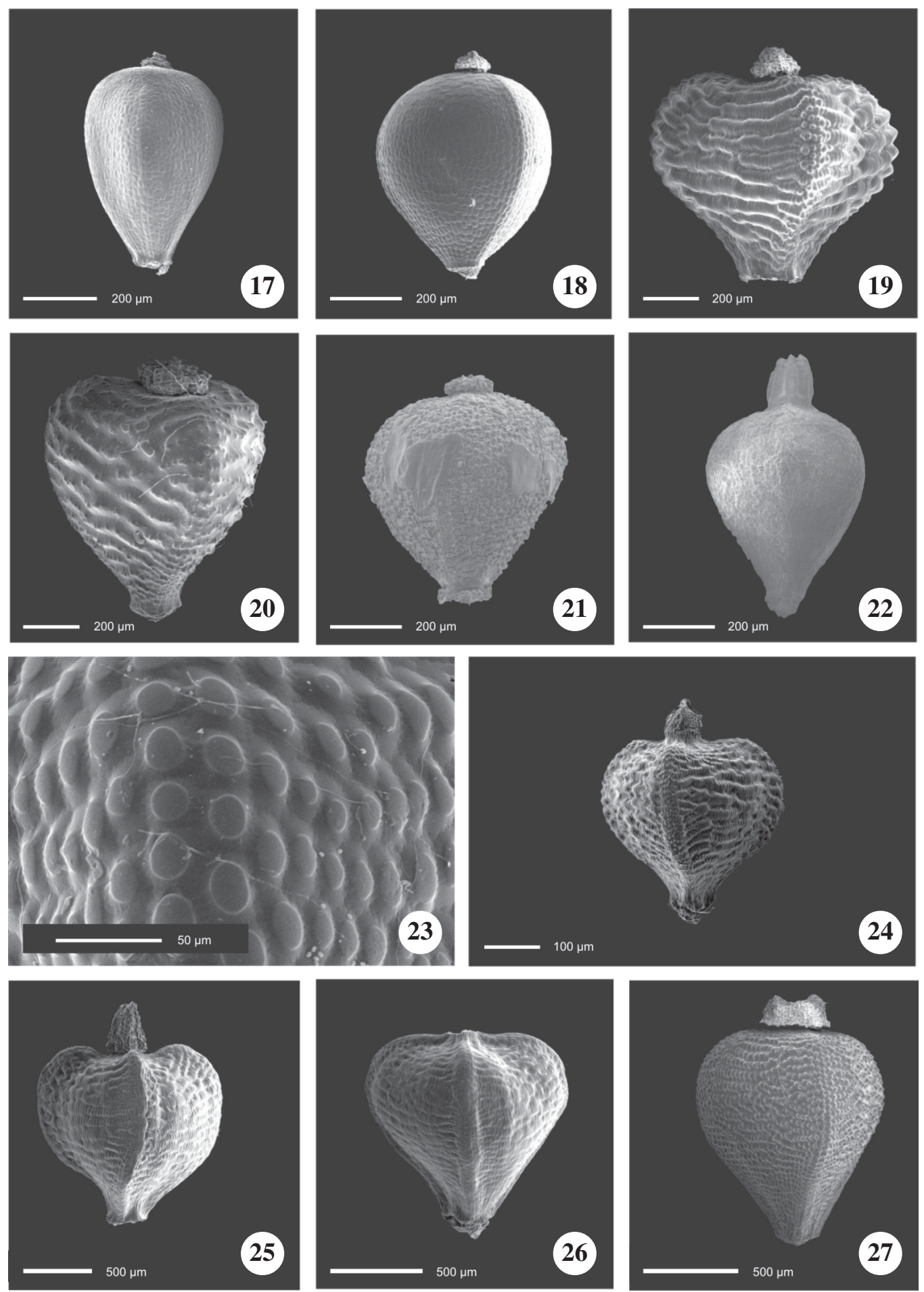

Figuras 17-27. Aquênio das espécies de Bulbostylis ao Microscópio Eletrônico de Varredura. 17. B. amabayensis. 18. B. tenuifolia. 19. B. conifera. 20. B. brevifolia. 21. B. jacobinae. 22. B. eleocharoides. 23. B. edwalliana. 24. B. lombardii. 25. B. emmerichiae. 26. B. sellowiana. 27. B. consanguinea.

Figure 17-27. Achene surface of Bulbostylis species to the Scanning Electron Microscope. 17. B. amabayensis. 18. B. tenuifolia. 19. B. conifera. 20. B. brevifolia. 21. B. jacobinae. 22. B. edwalliana. 23. B. eleocharoides. 24. B. lombardii. 25. B. emmerichiae. 26. B. sellowiana. 27. B. consanguinea. 
espécies com inflorescência uniespicada, duas apresentaram padrão transversalmente rugoso, duas tuberculado e uma reticulado. Entre as nove com inflorescência capitada, quatro apresentaram padrão transversalmente rugoso, um tuberculado e quatro reticulado. Por último, o grupo de espécies com padrão de inflorescência mais comum no gênero, as antelóides, apresentou como padrões mais frequentes: tuberculado, seguido pelo transversalmente rugoso e reticulado (tabela 2).

Corpos silicosos estão presentes nas espécies que exibem os padrões de ornamentação transversalmente rugoso e tuberculado (figuras 6, 8-11, 13, 14-16, 21, 22,27 ) apresentando, em geral, forma piramidal ou cônica e ausentes nas espécies com o padrão reticulado, (tabela 2). Corpos de sílica globosos foram detectados apenas em B. jacobinae e B. edwalliana (figuras 8, 23). Esta característica pode ser utilizada na identificação destes táxons.

Segundo Lye (2000), apesar do aquênio de muitas Cyperaceae possuir uma camada de cera cuticular formando uma superfície lisa ou mais ou menos brilhante, há muitas exceções. Além disso, ocasionalmente, a cera epicuticular presente nos frutos de Cyperaceae forma estruturas características de ornamentação incomuns. Pelo fato de não ter sido observada nenhuma diferença na ornamentação, a superfície do aquênio em Bulbostylis foi considerada lisa, exceto em $B$. jacobinae onde foi detectado a presença de placas de ceras epicuticulares (figura 21).

A maioria das espécies analisadas de Bulbostylis possui base do estilete persistente, sendo este um aspecto importante na caracterização genérica e na distinção de Fimbristylis, gênero semelhante morfologicamente mas que possui base do estilete decídua. Das espécies analisadas, apenas B. emmerichiae e B. sellowiana apresentam base do estilete decídua (figuras 25, 26). Além disso, foi observada a presença de sulco entre o ápice do aquênio e a base do estilete em $B$. consanguinea, B. emmerichiae, B. sellowiana e B. tenuifolia, podendo representar, possivelmente, um estágio intermediário entre base do estilete persistente e decídua (figuras 18 , 19, 27).

O comprimento do estilopódio também possui importância taxonômica, variando entre as espécies analisadas. Em 26 espécies estudadas, é 1/8 do comprimento do aquênio, seguido de $1 / 6 \mathrm{em}$ seis espécies e 1/4 em duas espécies. A base do estilete em B. eleocharoides é $1 / 3$ do comprimento do aquênio (figura 22), enquanto que em $B$. lombardii é $1 / 5$ (figura 24) e em B. jacobinae é 1/7 (figura 21).
A forma do estilopódio também variou entre as espécies: cilíndrico, triangular, umbonado, subcônico, subgloboso, plano e piramidal. Uma vez que não há variação da forma do estilopódio na mesma espécie, esta característica pode ser utilizada para a identificação.

Os caracteres micromorfológicos da superfície do aquênio discutidos aqui, em relação à escultura primária, foram semelhantes àqueles ressaltados por Barthlott (1984) e Menapace (1990) como úteis para a taxonomia das Cyperaceae. Além disso, destacamos a forma e comprimento do estilopódio em relação ao comprimento do aquênio, além da presença de estilopódio decíduo em duas espécies de Bulbostylis como bons caracteres adicionais na taxonomia do gênero.

Conclui-se que a análise micromorfológica da superfície do aquênio em espécies de Bulbostylis revelou caracteres de interesse taxonômico tais como: a variação da escultura primária com a proposição de três padrões de ornamentação: tuberculado, reticulado e transversalmente rugoso, sendo o primeiro encontrado com maior frequência nas espécies com inflorescência antelóide. Além disso, foi observado que a variação de alguns caracteres, tais como: a ornamentação das paredes anticlinais, a presença ou ausência de corpos silicosos e o contorno das células epidérmicas podem ser utilizados como auxílio na distinção entre as espécies do gênero.

Agradecimentos - Aos curadores dos herbários analisados. À Fapesp (Processo no. 99/03773-1), pela concessão da bolsa de doutorado à primeira autora. À Ana Claudia Araújo pelas sugestões no manuscrito.

\section{Referências bibliográficas}

ARAÚJO, A.C. \& LONGHI-WAGNER, H.M. 1997. Anatomia foliar e micromorfologia da superfície do fruto na taxonomia de Cyperus L. (Cyperaceae). Iheringia 48: 103-120.

BARROS, M. 1959. Dos Ciperaceas sudamericanas nuevas. Darwiniana 11:761-764.

BARTHLOTT, W. 1984. Microstrutural features of seed surfaces. In Current concepts in plant taxonomy (V.H. Heywood \& D. M. Moore, eds). London Academic Press, London, p.95-105.

DENTON, M.F. 1978. Taxonomic treatment of the "Luzulae" group of Cyperus. Contributions from the University of Michigan Herbarium 11:197-271.

DENTON, M.F. 1983. Anatomical studies of the "Luzulae" group of Cyperus (Cyperaceae). Systematic Botany 8:250-262.

ENDLICHER, S. 1836. Cyperaceae. Genera Plantarum secundum ordines naturales disposita, F. Beck, Vindobonae, p.109-118. 
GOVINDARAJALU, E. 1966. The systematic of South Indian Cyperaceae: Bulbostylis Kunth. Journal of Linnean Society 59:289-304.

HOLMGREN, P.K., HOLMGREN, N.H. \& BARNETT, L. G. 1990. Index Herbariorum. Part 1: the herbaria of the world. $8^{\text {th }}$ ed., New York Botanical Garden, New York.

KUKKONEN, I. 1967. Vegetative anatomy of Uncinia (Cyperaceae). Annals of Botany 31:523-544.

KUNTH, C.G. 1816. Nova Genera et Species Plantarum. Librariae Graeco-Latino-Germanico, Paris, v.1.

LÓPEZ, M.G., PRATA, A.P. \& THOMAS, W.W. 2007. New synonymy and new distributional records in Bulbostylis (Cyperaceae) from South America. Brittonia 59:88-96.

LYE, K. A. 1986. A new species of Abildgaardia (Cyperaceae) from Brasil. Nordic Journal of Botany 6:765-767.

LYE, K.A. 2000. Achene structure and function of structure in Cyperaceae. In Monocots: systematics and evolution (K.L. Wilson \& D.A. Morrison, eds). CSIRO Publishing, Melbourne, p.615-628.

MENAPACE, F.J. 1990. A preliminary micromorphological analysis of Eleocharis (Cyperaceae) achenes for systematic potential. Canadian Journal of Botany 69:1533-1541.

METCALFE, C.R. 1971. Anatomy of the monocotyledons, Cyperaceae. Vol. 5. Clarendon Press, Oxford.

NEES, C.G.D. 1834. Cyperaceengattungen. Linnaea 9:273535.

OLIVEIRA, E.C. 1980. Cyperaceae Juss. Morfologia dos aquênios de gêneros ocorrentes no Brasil. Rodriguésia 55:327-405.
PRATA, A.P., CAMELBEKE, K., REYNDERS, M., FÉDON, I.R. \& GOETGHEBEUR, P. 2007. Bulbostylis medusae (Cyperaceae) a new species from Venezuela. Novon 17: 67-71.

PRATA, A.P. 2004. O gênero Bulbostylis Kunth (Cyperaceae) no Brasil. Tese de doutorado, Universidade de São Paulo, São Paulo.

PERSOON, C.H. 1805. Synopsis Plantarum 1:57-65.

PERSOON, C.H. 1807. Synopsis Plantarum 2:534-547.

RITTER, M.R., MIOTTO, S.T.S. 2006. Micromorfologia da superfície do fruto de espécies de Mikania Willd. (Asteraceae) ocorrentes no Estado do Rio Grande do Sul, Brasil. Acta Botanica Brasilica 20:241-247.

SCHUYLER, A.E. 1971. Scanning electron microscopy of achene epidermis in species of Scirpus (Cyperaceae) and related genera. Proceedings of the Academy of Natural Sciences of Philadelphia 123:29-51.

STANDLEY, L.A. 1985. Systematics of the Acutae group of Carex (Cyperaceae) in the Pacific Northwest. Systematic Botany Monographs 7:1-106.

TOIVONEN, H. \& TIMONEN, T. 1976. Perigynium and achene epidermis in some species of Carex subg. Vignea (Cyperaceae), studied by scanning electron microscopy. Annales Botanici Fennici 13:49-59.

WATERWAY, M.J. 1990. Systematic implications of achene micromorphology in Carex section Hymenochlaenae (Cyperaceae). Canadian Journal of Botany 68:630-639.

ZECH, J.C. \& WUJEK, D.E. 1990. Scanning eletron microscopy of seeds in the taxonomy of Michigan Juncus. The Michigan Botanist 29:3-18. 